\title{
AdS black holes and finite $N$ indices
}

\author{
Prarit Agarwal, ${ }^{1, *}$ Sunjin Choi $\odot,{ }^{2, \dagger}$ Joonho Kim $\odot,{ }^{3, \star}$ Seok Kim, ${ }^{2, \S}$ and June Nahmgoong $\odot^{4, \|}$ \\ ${ }^{1}$ CRST and School of Physics and Astronomy, Queen Mary University of London, \\ London E1 4NS, United Kingdom \\ ${ }^{2}$ Department of Physics and Astronomy \& Center for Theoretical Physics, \\ Seoul National University, Seoul 08826, Korea \\ ${ }^{3}$ Institute for Advanced Study, Princeton, New Jersey 08540, USA \\ ${ }^{4}$ School of Physics, Korea Institute for Advanced Study, Seoul 02455, Korea
}

(Received 23 February 2021; accepted 27 April 2021; published 4 June 2021)

\begin{abstract}
We study the index of $4 \mathrm{~d} \mathcal{N}=4$ Yang-Mills theory with $U(N)$ gauge group, focusing on the physics of the dual Bogomolny-Prasad-Sommerfield black holes in $\operatorname{AdS}_{5} \times S^{5}$. Certain aspects of these black holes can be studied from finite $N$ indices with reasonably large $N^{2}$. We make numerical studies of the index for $N \leq 6$, by expanding it up to reasonably high orders in the fugacity. The entropy of the index agrees very well with the Bekenstein-Hawking entropy of the dual black holes, say at $N^{2}=25$ or 36 . Our data clarifies and supports the recent ideas which allowed analytic studies of these black holes from the index, such as the complex saddle points of the Legendre transformation and the oscillating signs in the index. In particular, the complex saddle points naturally explain the $\frac{1}{N}$-subleading oscillating patterns of the index. We also illustrate the universality of our ideas by studying a model given by the inverse of the MacMahon function.
\end{abstract}

DOI: 10.1103/PhysRevD.103.126006

\section{INTRODUCTION AND SUMMARY}

The superconformal index of large $N$ field theories [1-3] has recently received some attention [4-21] as it successfully accounts for the thermodynamic entropy of AdS black holes. In this paper, we want to present our numerical study of the $4 \mathrm{~d} \mathcal{N}=4$ superconformal index, showing that some aspects of the BPS (Bogomolny-Prasad-Sommerfield) black holes in $\mathrm{AdS}_{5}$ [22-25] can be investigated by numerically studying the index at finite $N$. Our numerical data will also nontrivially support certain recent ideas which enabled the analytic studies of these AdS black holes.

We define the Witten index of $4 \mathrm{~d} \mathcal{N}=4$ superconformal field theory on $S^{3} \times S^{1}$ as [2]

$$
Z\left(\Delta_{I}, \omega_{i}\right)=\operatorname{Tr}\left[(-1)^{F} e^{-\sum_{I=1}^{3} \Delta_{I} Q_{I}-\sum_{i=1}^{2} \omega_{i} J_{i}}\right]
$$

with the constraint $\Delta_{1}+\Delta_{2}+\Delta_{3}-\omega_{1}-\omega_{2}=0$ on the chemical potentials. $Q_{I}$ with $I=1,2,3$ denote the

\footnotetext{
*agarwalprarit@gmail.com

csj37100@snu.ac.kr

\#joonhokim@ias.edu

\$skim@phya.snu.ac.kr

"junenahmgoong@gmail.com
}

Published by the American Physical Society under the terms of the Creative Commons Attribution 4.0 International license. Further distribution of this work must maintain attribution to the author(s) and the published article's title, journal citation, and DOI. Funded by SCOAP.
$U(1)^{3} \subset S O(6)$ R-charges of $\mathcal{N}=4$ superalgebra, and $J_{i}$ with $i=1,2$ denote the $U(1)^{2} \subset S O(4)$ angular momenta on $S^{3}$. Only the BPS states with the energy $E=$ $\sum_{I=1}^{3} Q_{I}+\sum_{i=1}^{2} J_{i}$ can contribute to the index. Since the supersymmetric index is invariant under the continuous deformation of the gauge coupling, one can evaluate the index from the weakly interacting QFT. It can be done in a few steps. First, we obtain the following single-letter index [2]

$I_{\text {single }}\left(\Delta_{I}, \omega_{i}\right)=1-\frac{\left(1-e^{-\Delta_{1}}\right)\left(1-e^{-\Delta_{2}}\right)\left(1-e^{-\Delta_{3}}\right)}{\left(1-e^{-\omega_{1}}\right)\left(1-e^{-\omega_{2}}\right)}$

by counting all single-letter operators in the $\mathcal{N}=4$ vector multiplet that satisfy the above mentioned BPS energy condition. Next, we apply the Plethystic exponential to this index $I_{\text {single }}$ multiplied by the adjoint character $\chi_{\mathfrak{g}}(\mathbf{z})$ of the gauge algebra $\mathfrak{g},[2]$

$$
\begin{aligned}
& \operatorname{PE}\left[I_{\text {single }}\left(\Delta_{I}, \omega_{i}\right) \chi_{\mathfrak{g}}\left(z_{a}\right)\right] \\
& \quad \equiv \exp \left[\sum_{n=1}^{\infty} \frac{I_{\text {single }}\left(n \Delta_{I}, n \omega_{i}\right) \chi_{\mathfrak{g}}\left(z_{a}^{n}\right)}{n}\right] .
\end{aligned}
$$

Finally, we project to the set of gauge invariant states by integrating over $z_{a}$ with the Haar measure of the gauge group. The index of the $4 \mathrm{~d} \mathcal{N}=4$ theory with a gauge 
group corresponding to the Lie algebra $\mathfrak{g}$ reduces to a matrix model calculation giving the following integral [2]:

$$
Z_{\mathfrak{g}}=\oint d \mu_{\mathfrak{g}}(\mathbf{z}) \operatorname{PE}\left[I_{\text {single }}\left(\Delta_{I}, \omega_{i}\right) \chi_{\mathfrak{g}}(\mathbf{z})\right]
$$

Here $d \mu_{\mathfrak{g}}(\mathbf{z})$ is the Haar measure of $\mathfrak{g}$. Explicitly, it can be written as

$$
\begin{aligned}
\oint d \mu_{\mathfrak{g}}(\mathbf{z})= & \frac{1}{(2 \pi i)^{r}} \frac{1}{|W|} \oint_{\left|z_{1}\right|=1} \\
& \ldots \oint_{\left|z_{r}\right|=1} \frac{d z_{1} \ldots d z_{r}}{z_{1} \ldots z_{r}} \prod_{\alpha \in \Delta}\left(1-\mathbf{z}^{\alpha}\right),
\end{aligned}
$$

where $W$ is the Weyl group of $\mathfrak{g}, r$ is the rank, $z_{a}$ is the fugacity corresponding to its $a$-th Cartan generator and $\Delta$ is the set of its roots. It turns out that for numerical purposes it is more efficient to use a slightly modified definition of the Haar measure given by restricting the product in (1.5) to only the positive roots of $\mathfrak{g}$ [26]:

$$
\oint d \mu_{\mathfrak{g}}(\mathbf{z})=\frac{1}{(2 \pi i)^{r}} \oint_{\left|z_{1}\right|=1} \ldots \oint_{\left|z_{r}\right|=1} \frac{d z_{1} \ldots d z_{r}}{z_{1} \ldots z_{r}} \prod_{\alpha \in \Delta^{+}}\left(1-\mathbf{z}^{\alpha}\right) .
$$

This helps by removing the need to normalize the integral by the order of the Weyl group. From (1.4) and (1.2), $Z\left(\Delta_{I}, \omega_{i}\right)$ is invariant under $2 \pi i$ shift of each of $\Delta_{I}, \omega_{i}$. So one can equivalently study the index at the surface $\sum_{I} \Delta_{I}-\sum_{i} \omega_{i}=2 \pi i \mathbb{Z}$. Below, we shall often choose the right-hand side to be $2 \pi i$.

For our purposes it suffices to consider a special unrefined case of the above integral by setting $e^{-\Delta_{1}}=e^{-\Delta_{2}}=e^{-\Delta_{3}} \equiv e^{-\Delta}, e^{-\omega_{1}}=e^{-\omega_{2}} \equiv e^{-\omega}$. If one Legendre transforms to the microcanonical ensemble at macroscopic charges, this amounts to taking equal charges and equal angular momenta, $Q_{1}=Q_{2}=Q_{3}=Q$ and $J_{1}=J_{2}=J$. From $3 \Delta-2 \omega=2 \pi i \mathbb{Z}$, one can set $x^{2}=e^{-\Delta}, x^{3}=e^{-\omega}$ for certain $x$. The fugacity $x$ is now conjugate to the charge $j \equiv 6(Q+J)$. The expression in (1.4) then becomes

$$
Z_{\mathfrak{g}}=\oint d \mu_{\mathfrak{g}}(\mathbf{z}) \operatorname{PE}\left[\left(1-\frac{\left(1-x^{2}\right)^{3}}{\left(1-x^{3}\right)^{2}}\right) \chi_{\mathfrak{g}}(\mathbf{z})\right] .
$$

The resulting index can be expanded as

$$
Z=\sum_{j=0}^{\infty} \Omega_{j} x^{j}
$$

where $j \equiv 6(Q+J)$ and $\Omega_{j}$ are integers which count the number of BPS states (with -1 factor for fermions). For $U(N)$ gauge group, we shall study this index at $N=2,3,4$,
5,6 , by computing the coefficients of the fugacity expansion in $x$ up to fairly high orders, till $\mathcal{O}\left(x^{100}\right)$ for $N \leq 5$, and till $\mathcal{O}\left(x^{70}\right)$ for $N=6$. Naively, finite $N$ indices will be irrelevant for studying emergent gravitational phenomena expected in the large $N$ limit. In particular, one would like to study the large $N$ limit of $\Omega_{j}$ when $j$ is of order $N^{2} \gg 1$. In this limit, black hole like degeneracy will grow like $\log \left|\Omega_{i}\right| \sim N^{2}$ when $j \sim N^{2}$. Our starting point is that, in practice, taking $N=5$ or 6 has already large enough $N^{2}$, so that we can hope to see the black hole like exponential growth of $\Omega_{j}$ quite convincingly. In fact, plugging in $N^{2}=$ 25 or 36 to the geometric Bekenstein-Hawking entropy formula for the known $\mathrm{AdS}_{5}$ black holes, we shall find very good agreements with the field theory calculus of $\log \left|\Omega_{j}\right|$. In non-Abelian gauge theories, how small $\frac{1}{N}$ should be at finite $N$ to exhibit large $N$ behaviors depends on the type of physics one is interested in. So not too surprisingly, our finite $N$ approach does not clearly see certain types of black holes. For instance, we empirically find that the charge range for the so-called "small black holes" is not clearly resolved in our finite $N$ discretized analysis. (See Sec. II for more explanations.) The detailed physics that can be learned is outlined below, and will be elaborated more in Sec. III.

Our finite (but reasonably large) $N$ calculus reveals various interesting structures which shed more concrete lights on the recent analytic studies of these black holes. After computing the large $N$ free energy $\log Z$ as a function of chemical potentials $\Delta_{I}, \omega_{i}$, one makes a Legendre transformation to the microcanonical ensemble to compute the entropy. Legendre transformation is a saddle point approximation of the inverse Laplace transformation

$$
\Omega_{j}=\frac{1}{2 \pi i} \oint \frac{d x}{x} x^{-j} Z(x)
$$

at macroscopic charge $j$. (The formula can be generalized to refined $\Delta_{I}, \omega_{i}$, but we present the above unrefined formula for simplicity.) The fact is that the dominant saddle point values $x_{*}$ of $x$ (or $\Delta_{I}, \omega_{i}$ ) are complex, at real $j$ (or $Q_{I}$, $J_{i}$ ). The naively computed saddle point value of the integral, $\Omega_{j}\left(x_{*}\right) \equiv e^{S(j)}$, at real positive $j$ is therefore complex. Somewhat surprisingly, this simple fact apparently seems to have confused many people, leading to a number of $a d$ hoc prescriptions and interpretations on how to extract the correct physics out of this result. We stick to the natural interpretation of $[5,6,10]$ and find extremely nontrivial evidences supporting it from our numerical studies. We think this will confirm our interpretation to be the canonical picture, which goes as follows. From the unitarity of the underlying QFT, it is always guaranteed that one can find the complex conjugate saddle point $\bar{x}_{*}$ for any complex $x_{*}$. The conjugate saddle point value is given by $\Omega_{j}\left(\bar{x}_{*}\right)=e^{\overline{S(j)}}$. Adding the two equally dominant contributions, one obtains 


$$
\begin{aligned}
\Omega_{j} & \sim \Omega_{j}\left(x_{*}\right)+\Omega_{j}\left(\bar{x}_{*}\right) \\
& \sim \exp [\operatorname{Re}(S(j))+\cdots] \cos [\operatorname{Im}(S(j))+\cdots],
\end{aligned}
$$

where $\cdots$ denote possible subleading corrections at large $N^{2}$ and large $j$. (Note that $\operatorname{Re}(S(j))$ and $\operatorname{Im}(S(j))$ scale like $N^{2}$.) As will be manifest from our data in the next section, the integers $\Omega_{j}$ at macroscopic $j$ grow exponentially fast to account for the dual black holes, but come with possible minus signs at certain $j$ 's. Namely, $\Omega_{j}$ as a function of (quantized) $j$ oscillates between positive and negative integers as $j$ changes. However, the macroscopic Legendre transformation calculus is not sensitive to the precise quantized nature of $j$ and $\Omega_{j}$. Therefore, the best one can expect to see from this calculus is an exponentially growing envelope function, which is provided by $e^{\operatorname{Re}(S(j))}$, multiplied by a factor which oscillates between +1 and -1 , which is provided by $\cos [\operatorname{Im}(S(j))+\cdots]$ in the above expression.

Our numerical calculus will justify this interpretation. First, the computed entropy $\log \left|\Omega_{j}\right|$ from the integers $\Omega_{j}$ indeed takes the form of

$$
\operatorname{Re}(S(j))+\log [\cos (\operatorname{Im}(S(j)+\cdots)],
$$

where $\operatorname{Re}[S(j)]$ and $\operatorname{Im}[S(j)]$ are those computed recently from the index using various analytic methods (in the large $N$ and/or large charge limit). Furthermore, more importantly, investigating the overall signs in $\Omega_{j}$ from our numerical calculus, the sign oscillating pattern is also determined by the sign oscillation of $\cos (\operatorname{Im}(S(j))+\cdots)$, upon fitting a constant $\mathcal{O}(1)$ phase shift in "..." that has not yet been computed by any analytic methods. Therefore, a precise interpretation is given to $\operatorname{Im}(S(j))$, as containing the overall sign information of $\Omega_{j}$.

While comparing our numerically computed $\log \left|\Omega_{j}\right|$ with (1.11), confirming the appearance of the second term is nontrivial. This is because, while the first term is proportional to $N^{2}$, the second term is typically subleading because the macroscopic quantity $\operatorname{Im}(S(j)) \sim N^{2}$ is inside the cosine function. To detect the second term, it is crucial to make a precision computation of the index which sees this " $\frac{1}{N}$ corrections." Our finite $N$ indices (say at $N=5,6$ ) provide a perfect setup to confirm such structures, as these values of $N^{2}$ are large enough to provide a large $N$ hierarchy to various contributions to the entropy, while not being too large so that the subleading corrections are visible. We think our numerical support to the formula (1.11) is compelling. See Sec. III for the details.

The interpretations outlined above appear to be universal, which may appear in any indexlike generating functions that have negative integer coefficients at various orders. We illustrate that this is actually the case, by studying in detail the inverse of the MacMahon function

$$
\begin{aligned}
f(x) \equiv & \prod_{n=1}^{\infty}\left(1-x^{n}\right)^{n}=\sum_{j=0}^{\infty} \Omega_{j} x^{j}=1-x-2 x^{2}-x^{3} \\
& +0 x^{4}+4 x^{5}+4 x^{6}+7 x^{7}+3 x^{8}-2 x^{9} \\
& -9 x^{10}-17 x^{11}-\cdots
\end{aligned}
$$

At large $j$, one can analytically compute the macroscopic entropy given by $\log \left|\Omega_{j}\right| \sim \frac{3}{4}\left[2 \zeta(3) j^{2}\right]^{\frac{1}{3}}+\ldots$, where “..." denotes small $\frac{1}{j}$ corrections which can be concretely computed to any desired accuracy. On the other hand, $\Omega_{j}$ exhibits a characteristic oscillation between positive and negative integers. We shall illustrate that this is precisely realized in the Legendre transformation as the complex saddle points, where a formula like (1.11) will provide a perfect match. As we can explicitly compute the $\frac{1}{j}$ corrections to high orders, including the finite phase shifts in the second term of (1.11), our interpretation can be tested to very high accuracy in this model.

The remaining part of this paper is organized as follows. Section II summarizes our numerical results for the integers $\Omega_{j}$. We also explain some salient structures of the series $\Omega_{j}$, and also provide a comparison with the BekensteinHawking entropy of black holes. In Sec. III, we take a closer look at the structures of $\Omega_{j}$ and the $\frac{1}{N}$ correction, and provide various interpretations and discussions.

\section{NUMERICAL STUDY OF THE $\mathcal{N}=4$ INDEX}

We now specialize to the case of $4 \mathrm{~d} \mathcal{N}=4$ theories with a $U(N)$ gauge group. We would like to probe the regime

$$
Q_{I}, \quad J_{i} \sim N^{2} \gg 1
$$

However, the last inequality will be reasonably met by trying to take $N^{2}$ and charges to be as large as possible within our computational capability. We expand the index in $x$ (as introduced in Sec. I), perform the integral over $N$ variables on computer, to obtain various coefficients of

$$
Z_{U(N)}=\sum_{j=0}^{\infty} \Omega_{j} x^{j} \quad \text { with } \quad j \equiv 6(Q+J) .
$$

This is a straightforward exercise, with the main impediment coming from the availability of sufficient computing power. The computational-complexity of the integral grows extremely quickly as the rank of the gauge group increases. We were able to explicitly evaluate the above integral for $2 \leq N \leq 5$ up to $\mathcal{O}\left(x^{100}\right)$, as given in (A1)-(A4). For $U(6)$ we evaluated it up to $\mathcal{O}\left(x^{70}\right)$. The explicit expression of the $U(6)$ index is given by: 


$$
\begin{aligned}
Z_{U(6)}= & 1+3 x^{2}-2 x^{3}+9 x^{4}-6 x^{5}+21 x^{6}-18 x^{7}+48 x^{8}-42 x^{9}+99 x^{10}-96 x^{11} \\
& +200 x^{12}-198 x^{13}+345 x^{14}-340 x^{15}+540 x^{16}-426 x^{17}+564 x^{18}-234 x^{19} \\
& +189 x^{20}+636 x^{21}-1026 x^{22}+2262 x^{23}-2583 x^{24}+3438 x^{25}-1851 x^{26} \\
& -794 x^{27}+8757 x^{28}-20460 x^{29}+40398 x^{30}-63054 x^{31}+88401 x^{32} \\
& -99388 x^{33}+80856 x^{34}+4680 x^{35}-184576 x^{36}+494910 x^{37}-920943 x^{38} \\
& +1392360 x^{39}-1690101 x^{40}+1451568 x^{41}-114147 x^{42}-2931498 x^{43}+8129358 x^{44} \\
& -15183836 x^{45}+22398435 x^{46}-25748382 x^{47}+18439724 x^{48}+8645112 x^{49} \\
& -64166661 x^{50}+150570130 x^{51}-254339973 x^{52}+334069536 x^{53}-310532838 x^{54} \\
& +68770386 x^{55}+514459605 x^{56}-1501534768 x^{57}+2775637323 x^{58}-3887229606 x^{59} \\
& +3923925613 x^{60}-1520426502 x^{61}-4814089191 x^{62}+15863550944 x^{63} \\
& -30282658596 x^{64}+42802285428 x^{65}-42817602705 x^{66}+14831924490 x^{67} \\
& +57170104014 x^{68}-179436305580 x^{69}+331894244529 x^{70}+\mathcal{O}\left(x^{71}\right) .
\end{aligned}
$$

It was pointed out in [6] that the alternation of \pm signs of $\Omega_{j}$ demands special care when one attempts to extract it out at large $j$ using Legendre transformation. These sign alternations are generic: they also happen at lower $N$ 's. See the results in the Appendix A. We shall later observe more organized patterns of the sign alternations, as will be explained in Sec. III. Here, we simply note that the absolute degeneracy $\left|\Omega_{j}\right|$ indeed grows very fast at large $j$. For instance, one finds $\left|\Omega_{70}\right| \sim 3.3 \times 10^{11}$ at $N=6$, and $\left|\Omega_{100}\right| \sim 1.4 \times 10^{16}$ at $N=5$. We will see shortly that $\Omega_{j}$ grows quantitatively like the black hole entropy even at $N=5$, 6. See Fig. 1 for $\log \left|\Omega_{j}\right|$ and the signs of $\Omega_{j}$ at $N=5,6$.

We want to compare our indices at reasonably large $N$ with the spectra in the gravitational dual. At low energies, the BPS spectrum can be computed from the gas of gravitons [2]. A BPS graviton particle corresponds to a particular single trace BPS operator in the QFT dual. It is a valid approach when the energy $E$ satisfies $E \ll N$. In this limit, the BPS multigraviton states correspond to multitrace operators obtained by multiplying the above mentioned single trace operators, where one does not have to consider trace relations. As the energy grows, the finite $N$ effects of these graviton states have been studied in some detail in the BPS sector. The trace relations will start to enter from an energy of order $N$, reducing the number of independent operators than the naive multiparticle spectrum beyond this threshold. To see how this picture is reflected in our $\Omega_{j}$ 's, we first consider the index over BPS gravitons given by [2]

$$
\sum_{j=0}^{\infty} \Omega_{j}^{g} x^{j} \equiv \prod_{n=1}^{\infty} \frac{\left(1-x^{3 n}\right)^{2}}{\left(1-x^{2 n}\right)^{3}} .
$$

Comparing our $\Omega_{j}$ and $\Omega_{j}^{g}$, one finds that $\Omega_{j}=\Omega_{j}^{g}$ holds for $j \leq 2 N+1$. This can be seen exactly for all

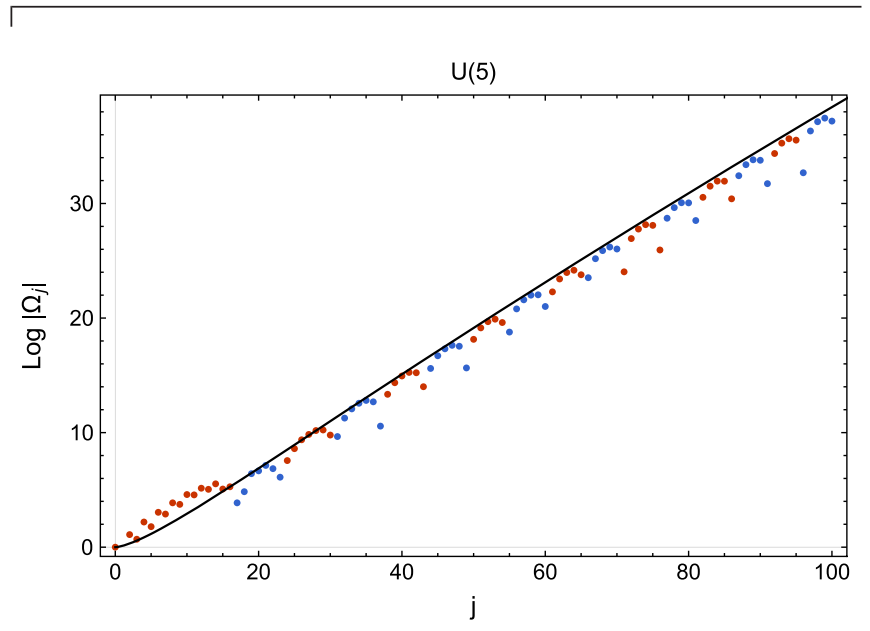

(a)

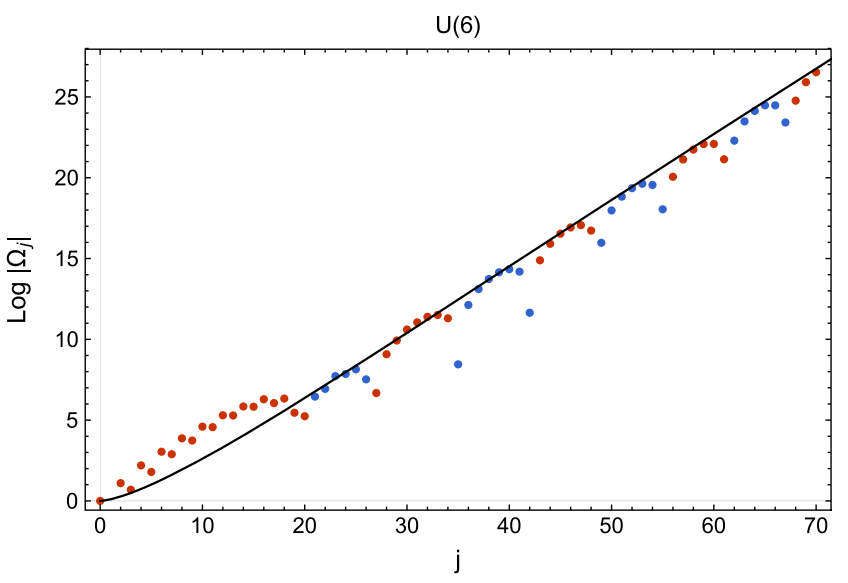

(b)

FIG. 1. Plots of $\log \left|\Omega_{j}\right|$ for $U(5)$ and $U(6)$ indices. The colors of the points encode the sign of $(-1)^{j} \Omega_{j}$ : red being positive and blue being negative. $\operatorname{Re}(S(j))$ computed from the black hole entropy function is the Bekenstein-Hawking entropy, given by the curve drawn with a solid black line. (a) $N=5$ and (b) $N=6$. 
$N=2, \ldots, 6$, and presumably holds exactly for other values of $N$. Slightly beyond this point, $j \gtrsim 2 N+1,\left|\Omega_{j}\right|$ is smaller than $\left|\Omega_{j}^{g}\right|$ for a certain while. So $j=2 N+1$ is naturally interpreted as the threshold where the trace relation starts to reduce the BPS states.

Now we consider the regime in which $j$ is substantially larger than this threshold, so that the resulting $\left|\Omega_{j}\right|$ cannot be explained from $\left|\Omega_{j}^{g}\right|$ with the trace relation reduction. $\left(\left|\Omega_{j}\right|\right.$ becomes bigger than $\left|\Omega_{j}^{g}\right|$ for sufficiently large $j$.) Eventually we enter a region with $j \sim N^{2}$, whose gravitational dual description will be the BPS black holes in AdS. To provide the comparison with the Bekenstein-Hawking entropy of these black holes, let us first explain the entropy function approach to understand its structures in a simple manner [27]. We present the results in the version which only keeps one fugacity $x[5,6]$. The entropy function we shall discuss assumes the convention $3 \Delta-2 \omega=2 \pi i$. Then $x^{2}=e^{-\Delta}, x^{3}=e^{-\omega}$ can be solved as

$$
x=e^{-\frac{\omega}{3}+\frac{2 \pi i}{3}}=-e^{-\frac{\Delta}{2}} .
$$

In this setup, consider the following entropy function of $j$ and $\omega$ :

$$
\begin{aligned}
S(\omega, j)= & \frac{N^{2} \Delta^{3}}{2 \omega^{2}}+\frac{\omega-2 \pi i}{3} j=\frac{N^{2}}{2 \omega^{2}}\left(\frac{2 \pi i+2 \omega}{3}\right)^{3} \\
& +\frac{\omega-2 \pi i}{3} j .
\end{aligned}
$$

The first term on the right-hand side originates from $\log Z$ in the grand canonical ensemble, and the second term is the Legendre transformation factor, whose exponential becomes $x^{-j}$ of (1.9). At fixed charge $j>0$, one extremizes $S(\omega, j)$ with $\omega$. This yields a cubic equation in $\omega$, which yields three different solutions $\omega_{*}$. Among these three, we take the one which yields maximal $\operatorname{Re}(S(j))>0$ where $S(j) \equiv S\left(\omega_{*}, j\right)$. At this solution, one finds

$$
\begin{aligned}
\omega_{*}= & -\xi \sqrt{\frac{3 \pi+3 \xi}{\pi-3 \xi}}+i \xi \\
j= & -\frac{N^{2}}{9} \frac{(\pi-2 \xi)^{2}(\pi+\xi)}{\xi^{3}} \\
\log Z= & +\frac{N^{2}}{18} \frac{\pi^{3}-9 \pi \xi^{2}-8 \xi^{3}}{\xi^{2}} \sqrt{\frac{\pi+\xi}{3 \pi-9 \xi}} \\
& -i \frac{N^{2}}{54} \frac{(\pi-8 \xi)(\pi+\xi)^{2}}{\xi^{2}},
\end{aligned}
$$

where $\xi$ is a real number satisfying $-\pi<\xi<0$. It parametrizes the imaginary part of $\omega$, and is a monotonically increasing function of $j$ implicitly given by the second line. Inserting this value back to $S(\omega, j)$, one obtains $S(j)$ given by

$$
\begin{aligned}
& \operatorname{Re}(S(j))=\frac{N^{2}}{6} \frac{\pi\left(\pi^{2}-2 \pi \xi-3 \xi^{2}\right)}{\xi^{2}} \sqrt{\frac{\pi+\xi}{3 \pi-9 \xi}} \\
& \operatorname{Im}(S(j))=-\frac{N^{2}}{18} \frac{\pi(\pi-5 \xi)(\xi+\pi)}{\xi^{2}}-\frac{2 \pi}{3} j
\end{aligned}
$$

where the relation $j(\xi)$ is assumed. The fact is that $\operatorname{Re}(S(j))$ is precisely the Bekenstein-Hawking entropy of the BPS AdS black holes of [22-24] at $Q \equiv Q_{1}=Q_{2}=Q_{3}$ and $J \equiv J_{1}=J_{2}$. More precisely, [22-24] found black hole solutions carrying two charges $Q, J$, depending on only one independent parameter. The entropy is a function of this parameter, which is in one to one correspondence with $j \equiv 6(Q+J)$. Therefore, expressing the one-parameter Bekenstein-Hawking entropy in terms of $j$, one obtains the above $\operatorname{Re}(S(j))$. Here, $N^{2}$ in the gravity side is related to the inverse Newton constant $G_{5}^{-1}$ of the $5 \mathrm{~d}$ gravity as $N^{2}=\frac{\pi \ell^{3}}{2 G_{5}}$, where $\ell$ is the radius of $\mathrm{AdS}_{5}$.

The classical gravity description will be reliable at small enough Newton constant, i.e., $N^{2} \gg 1$. To compare with our numerical results at $N=5,6$, we plug in $N^{2}=25$ or 36 to (2.8) expecting that $N^{2}$ is reasonably large. In Fig. 1, we have drawn these $\operatorname{Re}(S(j))$ by the black solid lines. At large enough charge $j$ (especially for $U(5)$ where we could do numerical calculations for larger charges), this agrees very well with the numerically computed entropy $\log \left|\Omega_{j}\right|$ of the index. There appear intriguing oscillations of our numerical $\log \left|\Omega_{j}\right|$, which appear to be subleading in $\frac{1}{N}$ at large enough charges. We shall comment on these subleading fluctuations in the next section.

Similar plots are shown for lower $N$ in the Appendix. Of course, inserting the finite values of $N^{2}$ to (2.8) becomes less meaningful for those lower values. As one can see from these figures, the numerical $\log \left|\Omega_{j}\right|$ and $\operatorname{Re}(S(j))$ do not agree that well for $N=2$ or $N=3$. Here we note that, although $S(j)$ of (2.8) is introduced here as the entropy function for the black hole, valid at $N^{2} \gg 1$, it has been shown [5] that (2.6) and (2.8) are true at any finite $N^{2}$ when $\omega$ becomes small (or equivalently, when $j \gg N^{2}$ ). This is called the "Cardy limit" of higher dimensional SCFTs in the recent literature. In this case, (2.8) and (2.6) have been derived from the field theory side for any value of $N$. As one can see gaps between $\log \left|\Omega_{j}\right|$ and $\operatorname{Re}(S(j))$ for $N=2$, 3 in Figs. 4 and 5 in Appendix A, it appears that the charge $j=100$ has not yet reached the Cardy regime.

We can also try to characterize which kinds of black holes are well described by our numerical data, and which kinds are not well visible. In AdS, one can classify black holes into "small black holes" and "large black holes" depending on various (closely related) criteria. The classification was originally made for AdS Schwarzschild black holes. However, similar notion exists for our BPS black holes by the charge playing the role of energy, and the inverse chemical potential playing the role of temperature. 
The most intuitive way to distinguish the AdS black holes is whether the "size" of the black hole is smaller than the AdS radius $\ell$, or larger than it. To make it more precise, consider the temperature $T$ of the black hole given by $\frac{1}{T}=\frac{d S(E)}{d E}$. For our BPS black holes, $\operatorname{Re}(\omega), j, \operatorname{Re}(S(j))$ play the role of $T^{-1}$, $E, S(E)$ respectively. They satisfy the analogous relation

$$
\frac{1}{3} \operatorname{Re}(\omega)=\frac{d[\operatorname{Re} S(j)]}{d j} .
$$

Now consider taking the second derivative with energy (or $j$ ),

$\frac{d T^{-1}(E)}{d E}=\frac{d^{2} S(E)}{d E^{2}}, \frac{1}{3} \frac{d \operatorname{Re}(\omega(j))}{d j}=\frac{d^{2}[\operatorname{Re} S(j)]}{d j^{2}}$,

where the first and second expressions apply for Schwarzschild black holes and our BPS black holes. The negativity of these expressions implies that the black holes are stable in the canonical and grand canonical ensemble, respectively, due to the heat capacity or susceptibility being positive. We call these black holes "large black holes." They are characterized by the entropy being a convex function of $E$ or $j$. Our BPS black holes are in the large black hole branch for $j>j_{0} \equiv \frac{(5+3 \sqrt{3}) N^{2}}{9}\left(\right.$ or $\left.-\frac{\pi}{\sqrt{3}}<\xi<0\right)$. On the other hand, for $j<j_{0}$ (or $-\pi<\xi<-\frac{\pi}{\sqrt{3}}$ ), the curve $S(j)$ is concave and one is in the small black hole branch. As one sees from the black curves in Fig. 1, the visibly concave region is at so small charges, that they are essentially overlapping with the region $j \leq 2 N+1$ in which the graviton description is good. Namely, we find that the small black hole branch squeezed by the graviton region from the left and $j_{0}$ from the right is not clearly visible from our finite $N$ indices. At large enough $N$, the two charge scales $j \sim 2 N+1$ and $j \sim j_{0}$ will be given enough hierarchy to allow a visible small black hole region. However, our finite $N$ index does not seem to have large enough $N$ to make this region clearly visible. Indeed, this can be clearly seen from our numerical plots in Fig. 1. In the small black hole region, $S(j)$ will increase very fast in $j$. However, our numerical $\log \left|\Omega_{j}\right|$ does not manifestly exhibit such an inflating region. It will be interesting to compute $\Omega_{j}$ 's for larger $N$ 's to see this region.

So far, we explained how to compare our $\log \left|\Omega_{j}\right|$ with $\operatorname{Re}(S(j))$ of the dual black holes. There is other interesting information that one can get from our numerical data, concerning $\operatorname{Im}(S(j))$, the signs of $\Omega_{j}$, and the subleading oscillations that one sees in the figures. These will be discussed in more detail in the next section.

\section{INTERPRETATIONS AND DISCUSSIONS}

In this section, we discuss more detailed information encoded in our numerical $\Omega_{j}$, and relate it to the interpretations made on (2.6).

We first study the signs of $\Omega_{j}$. The pattern of the signs visible in the series $Z(x)=\sum_{j} \Omega_{j} x^{j}$ apparently looks very complicated. However, one observes simplifications upon inserting $x \rightarrow-x$ :

$$
Z(-x)=\sum_{j}(-1)^{j} \Omega_{j} x^{j} .
$$

The signs of $(-1)^{j} \Omega_{j}$ are shown in Fig. 1 and also in the figures of Appendix A by the colors of the dots. After this substitution, one finds that the sign change pattern is correlated to the subleading oscillation pattern of $\log \left|\Omega_{j}\right|$. Namely, the sign changes only at the local minima of the oscillation.

At this point, we revisit the interpretation of complex $S(j)$ at the saddle point of the Legendre transformation at macroscopic charges, that we outlined in Sec. I. The interpretation asserts that the sign of $\cos [\operatorname{Im}(S(j))+\cdots]$ equals the sign of the integers $\Omega_{j}$. Since we have observed very simple sign oscillation patterns of our data $(-1)^{j} \Omega_{j}$, let us try to understand this also from the entropy function (2.6). Since $(-1)^{j}=e^{\pi i j}$, one finds that

$(-1)^{j} \Omega_{j} \sim \exp \left[\frac{N^{2}\left(\frac{2 \pi i}{3}+\frac{2 \omega_{*}}{3}\right)^{3}}{2 \omega_{*}^{2}}+\frac{\omega_{*}+\pi i}{3} j+\cdots\right]+$ c.c.,

where $\cdots$ are possible subleading corrections in small $\frac{1}{N^{2}}$ and $\frac{1}{j}$ that have not been computed to date. From this, one obtains

$(-1)^{j} \Omega_{j} \sim \exp [\operatorname{Re}(S(j))+\cdots] \cos [\operatorname{Im}(S(j))+\pi j+\cdots]$.

Although the subleading corrections to $\operatorname{Re}(S(j))$ will not affect our studies below, the corrections to $\operatorname{Im}(S(j))$ will be somewhat important since they will make a finite phase shift of the oscillation. The corresponding entropy (1.11) improving the black curve of Fig. 1 is shown in Fig. 2.

First, Fig. 2 clearly shows that the signs of $(-1)^{j} \Omega_{j}$ are equal to the sign of $\cos [\operatorname{Im}(S(j))+\pi j+\eta]$. As mentioned in the previous paragraph, we empirically fitted the possible subleading correction $\eta$ by an $\mathcal{O}(1)$ constant. Although $\eta$ is in principle a function of $j, N^{2}$ such as $\eta\left(\frac{j}{N^{2}}\right)$, constant $\eta$ seems to be reasonably good within the relatively short ranges of charges in Fig. 2. ${ }^{1}$ The agreements in Fig. 2 justify our interpretation that the oscillation caused by the complex saddle point accounts for the sign oscillations of $\Omega_{j}$. Moreover, Fig. 2 shows that the oscillation of $|\cos [\operatorname{Im}(S(j))+\pi j+\eta]|$ accounts for the subleading oscillations of our numerically computed $\log \left|\Omega_{j}\right|$. Therefore, we

\footnotetext{
${ }^{1}$ We also note that, upon including the 1-loop determinant factor of the Legendre transformation (2.6) in this framework, one obtains much better agreements than those in Fig. 2. However, we do not show these results here since they do not seem to be based on a systematic calculus of the subleading terms.
} 


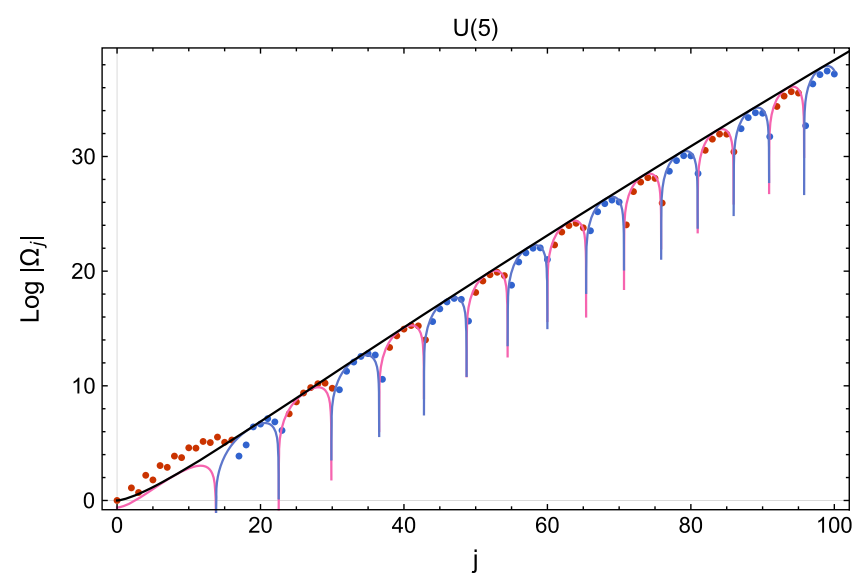

(a)

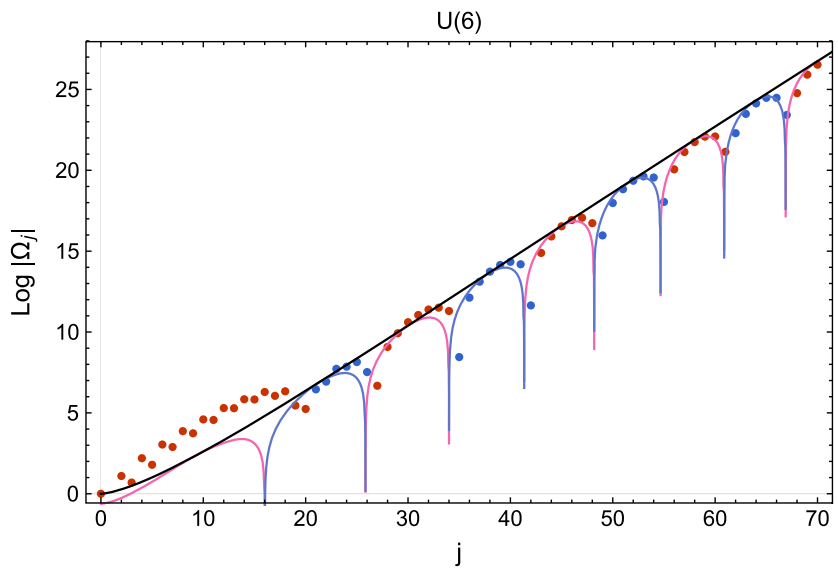

(b)

FIG. 2. Same plots as Fig. 1, with the extra red/blue curves for $\operatorname{Re}(S(j))+\log |\cos [\operatorname{Im}(S(j))+\pi j+\eta]|$. A subleading constant $\eta$ is empirically tuned to $\eta \approx-1$ to minimize the overall offphase behaviors. The red and blue colors of the curves denote $\cos [\operatorname{Im}(S(j))+\pi j+\eta] \gtrless 0$, respectively. (a) $N=5$ and (b) $N=6$.

find that our finite $N$ numerical data strongly supports the detailed structures of the macroscopic entropy computed at the complex saddle points of Legendre transformation.

As mentioned in the Introduction, it seems that our interpretation for the complex saddle point is very universal. To confirm this expectation, it will be helpful to study other indexlike generating functions which are simpler than the large $N$ index of the $\mathcal{N}=4$ Yang-Mills theory. In particular, for the Yang-Mills index, note that the analytic form of $S(j)$ is known only to the leading order in large $N$ and $j$. Due to this limitation, we added an empirical constant $\eta$ at a subleading order to see if the structures of $S(j)$ and $\Omega_{j}$ are compatible with each other. So it will be desirable to study simpler examples in which we can easily compute the subleading corrections for the precision tests.

As a simple example, consider the inverse of the MacMahon function, $f(x)=\prod_{n=1}^{\infty}\left(1-x^{n}\right)^{n}=\exp \left[-\sum_{n=1}^{\infty} \frac{1}{n} \frac{x^{n}}{\left(1-x^{n}\right)^{2}}\right] \equiv \sum_{j=0}^{\infty} \Omega_{j} x^{j}$.

Numerically, one can easily expand $f(x)$ in power series of $x$ with a computer to very high orders. At large charge $j$, one can see that the resulting $\Omega_{j}$ 's become macroscopic with sign oscillations. We shall now make an analytic evaluation of the asymptotic entropy at $j \gg 1$, with necessary subleading corrections in $\frac{1}{j}$ included. We would like to compute

$$
\begin{aligned}
\Omega_{j} & =\frac{1}{2 \pi i} \oint \frac{d x}{x} x^{-j} f(x) \\
& =\frac{1}{2 \pi i} \oint \frac{d x}{x} \exp \left[j \beta-\sum_{n=1}^{\infty} \frac{1}{n} \frac{e^{-n \beta}}{\left(1-e^{-n \beta}\right)^{2}}\right]
\end{aligned}
$$

where $x \equiv e^{-\beta}$. The saddle point values $\beta_{*}$ of $\beta$ will be small complex numbers with $\operatorname{Re}\left(\beta_{*}\right)>0$. At small $\beta$, one can use

$$
\begin{aligned}
-\sum_{n=1}^{\infty} \frac{1}{n} \frac{e^{-n \beta}}{\left(1-e^{-n \beta}\right)^{2}}= & -\frac{\zeta(3)}{\beta^{2}}-\frac{1}{12} \log \beta-\zeta^{\prime}(-1,0)+\frac{\beta^{2}}{2880} \\
& +\frac{\beta^{4}}{725760}+\frac{\beta^{6}}{43545600}+\cdots,
\end{aligned}
$$

where $\zeta(s)$ is the Riemann zeta function, and $\zeta^{\prime}(-1,0) \approx$ -0.165421 is the derivative $\zeta^{\prime}(s, q) \equiv \frac{\partial \zeta(s, q)}{\partial s}$ of the Hurwitz zeta function. Using this formula with higher order corrections in small $\beta$, one can approximate the integral (3.5) with subleading corrections in $\frac{1}{j}$ included. One finds that the following mutually complex conjugate pair of saddle points are dominant:

$\beta_{*}^{ \pm}=e^{ \pm \frac{\pi i}{3}}\left(\frac{2 \zeta(3)}{j}\right)^{\frac{1}{3}}+\frac{1}{36 j}+\frac{e^{\mp \frac{\pi i}{3}}}{1296\left(2 \zeta(3) j^{5}\right)^{\frac{1}{3}}}+\cdots$

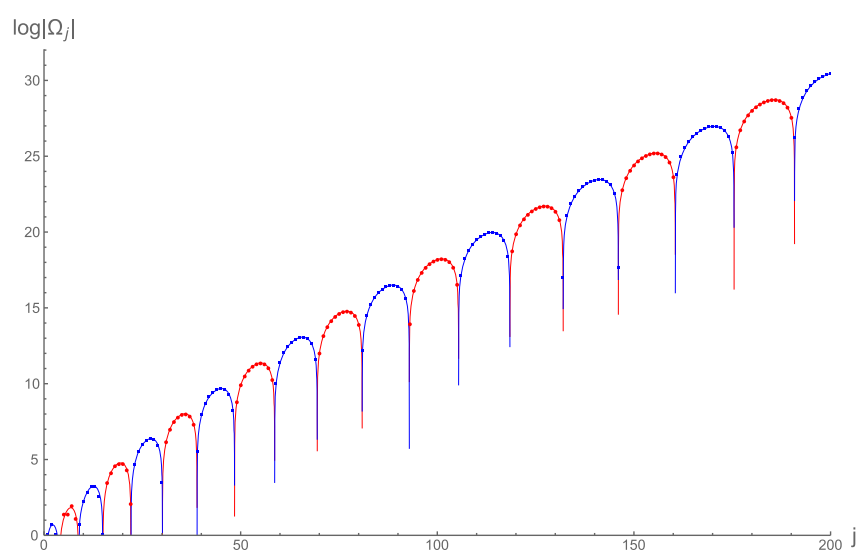

FIG. 3. Two plots of $\log \left|\Omega_{j}\right|$ for the MacMahon function. Red/ blue colors denote the positive/negative signs of $\Omega_{j}$. 
Performing the Gaussian approximations at these two saddle points (with some subleading terms included) and adding the two contributions, one obtains

$$
\begin{aligned}
\Omega_{j} \sim & \frac{1}{(2 \pi)^{\frac{1}{2}}} \sum_{ \pm} \exp \left[\frac{3}{2} e^{ \pm \frac{\pi i}{3}}\left(2 \zeta(3) j^{2}\right)^{\frac{1}{3}}+\frac{1}{36} \log j-\zeta^{\prime}(-1,0)-\frac{\log (2 \zeta(3))}{36} \mp \frac{\pi i}{36}+\cdots\right] \\
& \times\left[3 e^{\mp \frac{\pi i}{3}}\left(\frac{j^{4}}{2 \zeta(3)}\right)^{\frac{1}{3}}+\frac{1}{4} e^{ \pm \frac{\pi i}{3}}\left(\frac{j}{2 \zeta(3)}\right)^{\frac{2}{3}}-\frac{1}{216 \zeta(3)}+\cdots\right]^{-\frac{1}{2}} \cdot[1+\cdots] .
\end{aligned}
$$

Here, the three factors on the right-hand side come from the saddle point action, the 1-loop determinant, and possible higher loop corrections, respectively. We plot this asymptotic $\log \left|\Omega_{j}\right|$ in Fig. 3, together with the dotted plot obtained from the series expansion up to $\mathcal{O}\left(x^{200}\right)$ order.

\section{ACKNOWLEDGMENTS}

We thank Ashoke Sen for suggesting us to study the finite $N$ indices to better understand AdS black holes, which was the starting point of this project. We also thank Shota Komatsu and Xi Yin for the helpful comments and questions. P. A., S. C., and S. K. are supported in part by the National Research Foundation of Korea (NRF) Grant No. 2018R1A2B6004914. The work of P. A. is also supported in part by the Korea Research Fellowship Program through the National Research Foundation of Korea funded by the Ministry of Science, ICT and Future Planning, Grant No. 2016H1D3A1938054 and in part by the Royal Society Research Fellows Enhancement Award, Grant No. RGFIEA 1181049. The work of SC is also supported by NRF-2017Global Ph.D. Fellowship Program. J. K. is supported by the NSF Grant No. PHY-1911298. J. N. is supported by a KIAS Individual Grant No. PG76401. S. K. is also supported in part by the National Research Foundation of Korea (NRF) Grant No. 2021R1A2C2012350.

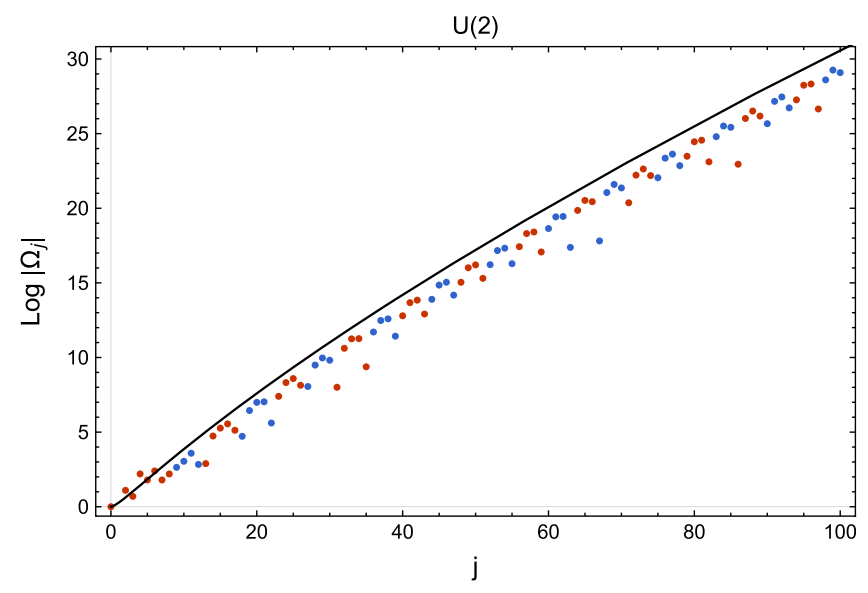

FIG. 4. $\quad N=2$
Note added.-On the same day this work appeared on the arXiv, the paper [28] was also on the arXiv, which overlaps with our Sec. II.

\section{APPENDIX: NUMERICAL DATA}

Here we collect the numerical expression for the $U(N)$ index with $2 \leq N \leq 5$. The following expression is the $U(2)$ index, which is illustrated in Fig. 4.

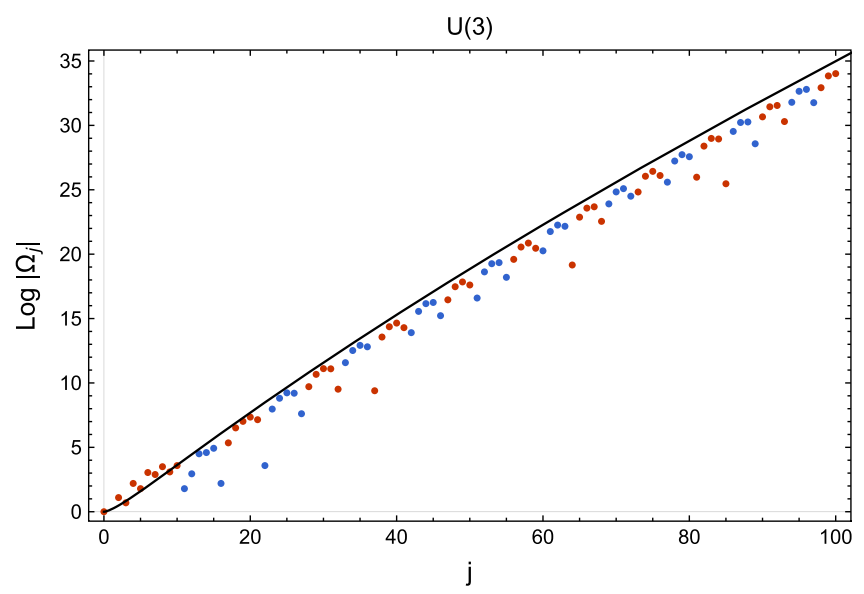

FIG. 5. $\quad N=3$

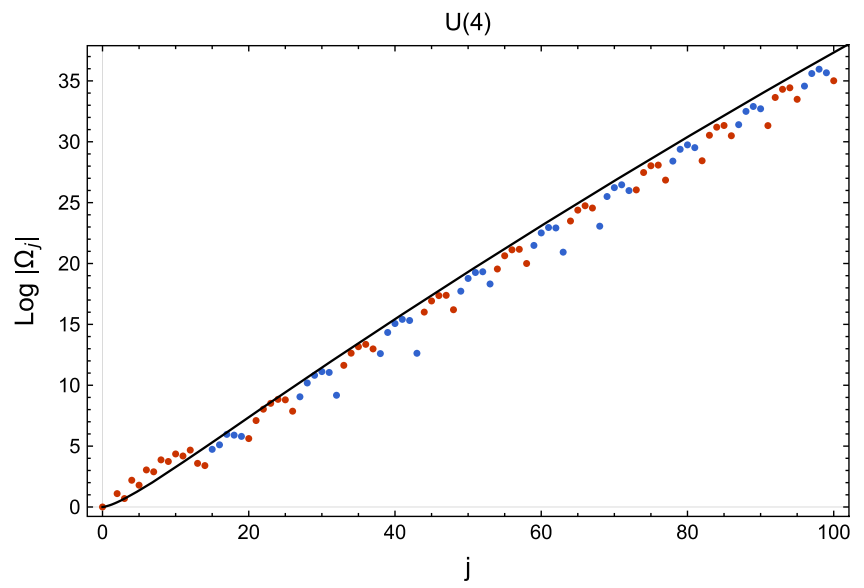

FIG. 6. $\quad N=4$ 


$$
\begin{aligned}
Z_{U(2)}= & 1+3 x^{2}-2 x^{3}+9 x^{4}-6 x^{5}+11 x^{6}-6 x^{7}+9 x^{8}+14 x^{9}-21 x^{10}+36 x^{11}-17 x^{12} \\
& -18 x^{13}+114 x^{14}-194 x^{15}+258 x^{16}-168 x^{17}-112 x^{18}+630 x^{19}-1089 x^{20} \\
& +1130 x^{21}-273 x^{22}-1632 x^{23}+4104 x^{24}-5364 x^{25}+3426 x^{26}+3152 x^{27} \\
& -13233 x^{28}+21336 x^{29}-18319 x^{30}-2994 x^{31}+40752 x^{32}-76884 x^{33}+78012 x^{34} \\
& -11808 x^{35}-121384 x^{36}+262206 x^{37}-293145 x^{38}+91904 x^{39}+359775 x^{40} \\
& -867906 x^{41}+1026540 x^{42}-404454 x^{43}-1086312 x^{44}+2815744 x^{45}-3415932 x^{46} \\
& +1436112 x^{47}+3403791 x^{48}-9007578 x^{49}+10895604 x^{50}-4420644 x^{51}-11068260 x^{52} \\
& +28481682 x^{53}-33440475 x^{54}+11822670 x^{55}+36950502 x^{56}-88878842 x^{57} \\
& +98770059 x^{58}-25918986 x^{59}-124747447 x^{60}+272655942 x^{61}-279580701 x^{62} \\
& +35207790 x^{63}+419441625 x^{64}-818211192 x^{65}+751976333 x^{66}+54317328 x^{67} \\
& -1386833514 x^{68}+2387940758 x^{69}-1893048381 x^{70}-700663056 x^{71}+4467470232 x^{72} \\
& -6731222448 x^{73}+4333120557 x^{74}+3746183998 x^{75}-13926217512 x^{76}+18169226454 x^{77} \\
& -8426843619 x^{78}-15799669950 x^{79}+41774162736 x^{80}-46405515308 x^{81} \\
& +10894454985 x^{82}+58624684746 x^{83}-119915881179 x^{84}+110030518596 x^{85} \\
& +9268878210 x^{86}-198813575484 x^{87}+327212977320 x^{88}-233510264916 x^{89} \\
& -140308837617 x^{90}+626333831526 x^{91}-840626319591 x^{92}+404682823524 x^{93} \\
& +692617559553 x^{94}-1844851526580 x^{95}+2001340988797 x^{96}-375443664666 x^{97} \\
& -2639017467255 x^{98}+5082041971496 x^{99}-4283590699023 x^{100}+\mathcal{O}\left(x^{101}\right)
\end{aligned}
$$

The next expression is the $U(3)$ index, whose $\log \left|\Omega_{j}\right|$ is drawn in Fig. 5 .

$$
\begin{aligned}
Z_{U(3)}= & 1+3 x^{2}-2 x^{3}+9 x^{4}-6 x^{5}+21 x^{6}-18 x^{7}+33 x^{8}-22 x^{9}+36 x^{10}+6 x^{11}-19 x^{12}+90 x^{13} \\
& -99 x^{14}+138 x^{15}-9 x^{16}-210 x^{17}+672 x^{18}-1116 x^{19}+1554 x^{20}-1270 x^{21}-36 x^{22} \\
& +2898 x^{23}-6705 x^{24}+10224 x^{25}-9918 x^{26}+2018 x^{27}+16470 x^{28}-42918 x^{29} \\
& +66906 x^{30}-66006 x^{31}+13566 x^{32}+106404 x^{33}-273204 x^{34}+407442 x^{35}-364710 x^{36} \\
& -12024 x^{37}+778272 x^{38}-1731542 x^{39}+2300499 x^{40}-1611774 x^{41}-1093848 x^{42} \\
& +5702562 x^{43}-10400586 x^{44}+11407626 x^{45}-4086693 x^{46}-13996782 x^{47} \\
& +38712766 x^{48}-56127654 x^{49}+44316099 x^{50}+16085226 x^{51}-122617179 x^{52} \\
& +231054624 x^{53}-251544720 x^{54}+80412606 x^{55}+324099348 x^{56}-844286204 x^{57} \\
& +1147990887 x^{58}-767030682 x^{59}-628392075 x^{60}+2808255348 x^{61}-4642468821 x^{62} \\
& +4223264234 x^{63}+209141406 x^{64}-8584019040 x^{65}+17327115906 x^{66} \\
& -19194283332 x^{67}+6197598675 x^{68}+24052600650 x^{69}-61026825105 x^{70} \\
& +78594793644 x^{71}-43722790228 x^{72}-60628872366 x^{73}+205754044713 x^{74} \\
& -300949636742 x^{75}+217767461283 x^{76}+129914189388 x^{77}-671070962823 x^{78} \\
& +1099745830260 x^{79}-937888762842 x^{80}-191081792160 x^{81}+2135620393074 x^{82} \\
& -3884644088484 x^{83}+3715774679244 x^{84}-114903322902 x^{85}-6683223253806 x^{86} \\
& +13381744369680 x^{87}-13925733216507 x^{88}+2562254228766 x^{89} \\
& +20719792872015 x^{90}-45245335312008 x^{91}+50127612882930 x^{92}
\end{aligned}
$$




$$
\begin{aligned}
& -14402257204784 x^{93}-64103402035710 x^{94}+150872971344750 x^{95} \\
& -174917721819708 x^{96}+62316941736600 x^{97}+199276922573595 x^{98} \\
& -497907763520398 x^{99}+595172510765379 x^{100}+\mathcal{O}\left(x^{101}\right)
\end{aligned}
$$

The $U(4)$ index comes next. The corresponding figure, $\log \left|\Omega_{j}\right|$ vs $j$, is drawn in Fig. 6.

$$
\begin{aligned}
Z_{U(4)}= & 1+3 x^{2}-2 x^{3}+9 x^{4}-6 x^{5}+21 x^{6}-18 x^{7}+48 x^{8}-42 x^{9}+78 x^{10}-66 x^{11}+107 x^{12} \\
& -36 x^{13}+30 x^{14}+114 x^{15}-165 x^{16}+390 x^{17}-366 x^{18}+330 x^{19}+276 x^{20}-1212 x^{21} \\
& +3081 x^{22}-4986 x^{23}+6924 x^{24}-6654 x^{25}+2616 x^{26}+8528 x^{27}-26571 x^{28} \\
& +49800 x^{29}-67651 x^{30}+63096 x^{31}-9678 x^{32}-112980 x^{33}+307098 x^{34}-522066 x^{35} \\
& +634029 x^{36}-436260 x^{37}-296460 x^{38}+1682020 x^{39}-3497613 x^{40}+4937946 x^{41} \\
& -4501122 x^{42}+304512 x^{43}+8971113 x^{44}-22380734 x^{45}+34738953 x^{46} \\
& -35553996 x^{47}+10888602 x^{48}+49956294 x^{49}-142303191 x^{50}+231744000 x^{51} \\
& -246464136 x^{52}+90402078 x^{53}+309123032 x^{54}-917051802 x^{55}+1494916050 x^{56} \\
& -1558557796 x^{57}+485393061 x^{58}+2144544540 x^{59}-5983505013 x^{60} \\
& +9333423798 x^{61}-9004631841 x^{62}+1231871108 x^{63}+15915475365 x^{64} \\
& -38937814944 x^{65}+55770600072 x^{66}-46223256036 x^{67}-10405285128 x^{68} \\
& +118932061824 x^{69}-247095009891 x^{70}+311970699564 x^{71}-193686936205 x^{72} \\
& -205315072914 x^{73}+855723695370 x^{74}-1490314195506 x^{75}+1572823900839 x^{76} \\
& -458786822988 x^{77}-2181976709955 x^{78}+5759182587780 x^{79}-8289856609587 x^{80} \\
& +6601945579040 x^{81}+2245784042823 x^{82}-18254661918174 x^{83}+35440988310091 x^{84} \\
& -40697268408630 x^{85}+17515834035681 x^{86}+43558153249536 x^{87} \\
& -129719118983523 x^{88}+194052483593046 x^{89}-160650745697554 x^{90} \\
& -40311995227758 x^{91}+407070606690366 x^{92}-795660945732754 x^{93} \\
& +899816226757623 x^{94}-349806028105302 x^{95}-1035026648995290 x^{96} \\
& +2903482927460364 x^{97}-4145273582018487 x^{98}+3091519137195862 x^{99} \\
& +1604158693277994 x^{100}+\mathcal{O}\left(x^{101}\right)
\end{aligned}
$$

The following series expression is the $U(5)$ index. The relevant plot of $\log \left|\Omega_{j}\right|$ is given in Fig. 1(a).

$$
\begin{aligned}
Z_{U(5)}= & 1+3 x^{2}-2 x^{3}+9 x^{4}-6 x^{5}+21 x^{6}-18 x^{7}+48 x^{8}-42 x^{9}+99 x^{10}-96 x^{11}+172 x^{12} \\
& -156 x^{13}+252 x^{14}-160 x^{15}+195 x^{16}+48 x^{17}-127 x^{18}+612 x^{19}-783 x^{20}+1258 x^{21} \\
& -948 x^{22}+450 x^{23}+1921 x^{24}-5430 x^{25}+11793 x^{26}-18812 x^{27}+26379 x^{28}-27750 x^{29} \\
& +17809 x^{30}+15648 x^{31}-78324 x^{32}+175030 x^{33}-285576 x^{34}+366024 x^{35}-323807 x^{36} \\
& +38856 x^{37}+624894 x^{38}-1718016 x^{39}+3094992 x^{40}-4226862 x^{41}+4098270 x^{42} \\
& -1210728 x^{43}-5968935 x^{44}+18061488 x^{45}-33152565 x^{46}+44941584 x^{47}-41448422 x^{48} \\
& +6241896 x^{49}+75761478 x^{50}-205993284 x^{51}+354209109 x^{52}-440168670 x^{53} \\
& +328572109 x^{54}+142704804 x^{55}-1079522706 x^{56}+2385844062 x^{57}-3584202447 x^{58} \\
& +3694263972 x^{59}-1331772481 x^{60}-4771857420 x^{61}+14697077445 x^{62}-25833114276 x^{63} \\
& +31549909440 x^{64}-21264664440 x^{65}-16439430686 x^{66}+86286819246 x^{67}
\end{aligned}
$$




$$
\begin{aligned}
& -174750537792 x^{68}+238416590234 x^{69}-201108631665 x^{70}-27442949994 x^{71} \\
& +499854484406 x^{72}-1146580228470 x^{73}+1684959423831 x^{74}-1584800711048 x^{75} \\
& +184556608692 x^{76}+2953939765242 x^{77}-7447464688605 x^{78}+11432006505378 x^{79} \\
& -11287805022885 x^{80}+2416173603110 x^{81}+18314405974467 x^{82}-48439160197746 x^{83} \\
& +75397207473690 x^{84}-74801457474012 x^{85}+16057846263102 x^{86}+120661512888900 x^{87} \\
& -316568078311605 x^{88}+485306430414990 x^{89}-464824039417731 x^{90} \\
& +60350744120262 x^{91}+837845036799732 x^{92}-2071759782098082 x^{93} \\
& +3041713804417725 x^{94}-2691482911939584 x^{95}-156200831519985 x^{96} \\
& +5991608828442690 x^{97}-13462930267605216 x^{98}+18424199416716136 x^{99} \\
& -14187219139048212 x^{100}+\mathcal{O}\left(x^{101}\right)
\end{aligned}
$$

[1] C. Romelsberger, Counting chiral primaries in $N=1$, $d=4$ superconformal field theories, Nucl. Phys. B747, 329 (2006).

[2] J. Kinney, J. M. Maldacena, S. Minwalla, and S. Raju, An Index for 4 dimensional super conformal theories, Commun. Math. Phys. 275, 209 (2007).

[3] J. Bhattacharya, S. Bhattacharyya, S. Minwalla, and S. Raju, Indices for superconformal field theories in 3, 5 and 6 dimensions, J. High Energy Phys. 02 (2008) 064.

[4] A. Cabo-Bizet, D. Cassani, D. Martelli, and S. Murthy, Microscopic origin of the Bekenstein-Hawking entropy of supersymmetric $\mathrm{AdS}_{5}$ black holes, J. High Energy Phys. 10 (2019) 062.

[5] S. Choi, J. Kim, S. Kim, and J. Nahmgoong, Large AdS black holes from QFT, arXiv:1810.12067.

[6] S. Choi, J. Kim, S. Kim, and J. Nahmgoong, Comments on deconfinement in AdS/CFT, arXiv:1811.08646.

[7] F. Benini and P. Milan, Black Holes in $4 \mathrm{~d} \mathcal{N}=4$ SuperYang-Mills, Phys. Rev. X 10, 021037 (2020).

[8] M. Honda, Quantum black hole entropy from 4d supersymmetric cardy formula, Phys. Rev. D 100, 026008 (2019).

[9] A. Arabi Ardehali, Cardy-like asymptotics of the $4 \mathrm{~d} \mathcal{N}=4$ index and $\mathrm{AdS}_{5}$ blackholes, J. High Energy Phys. 06 (2019) 134.

[10] S. Choi and S. Kim, Large $\mathrm{AdS}_{6}$ black holes from $\mathrm{CFT}_{5}$, arXiv:1904.01164.

[11] J. Kim, S. Kim, and J. Song, A $4 d \quad N=1$ cardy formula, J. High Energy Phys. 01 (2021) 025.

[12] A. Cabo-Bizet, D. Cassani, D. Martelli, and S. Murthy, The asymptotic growth of states of the $4 \mathrm{~d} \mathcal{N}=1$ superconformal index, J. High Energy Phys. 08 (2019) 120.

[13] A. Amariti, I. Garozzo, and G. Lo Monaco, Entropy function from toric geometry, arXiv:1904.10009.

[14] G. Kántor, C. Papageorgakis, and P. Richmond, AdS 7 blackhole entropy and 5D $\mathcal{N}=2$ Yang-Mills, J. High Energy Phys. 01 (2020) 017.
[15] J. Nahmgoong, 6d superconformal Cardy formulas, J. High Energy Phys. 02 (2021) 092.

[16] A. González Lezcano and L. A. Pando Zayas, Microstate counting via Bethe Ansätze in the $4 \mathrm{~d} \mathcal{N}=1$ superconformal index, J. High Energy Phys. 03 (2020) 088.

[17] S. Choi, C. Hwang, and S. Kim, Quantum vortices, M2-branes and black holes, arXiv:1908.02470.

[18] S. Choi and C. Hwang, Universal 3d cardy block and black hole entropy, J. High Energy Phys. 03 (2020) 068.

[19] A. A. Ardehali, J. Hong, and J. T. Liu, Asymptotic growth of the $4 \mathrm{~d} \mathcal{N}=4$ index and partially deconfined phases, J. High Energy Phys. 07 (2020) 073.

[20] A. Cabo-Bizet and S. Murthy, Supersymmetric phases of $4 \mathrm{~d} \mathcal{N}=4 \mathrm{SYM}$ at large $N$, J. High Energy Phys. 09 (2020) 184.

[21] P. M. Crichigno and D. Jain, The 5d superconformal index at large $N$ and black holes, J. High Energy Phys. 09 (2020) 124.

[22] J. B. Gutowski and H. S. Reall, Supersymmetric AdS(5) black holes, J. High Energy Phys. 02 (2004) 006.

[23] J. B. Gutowski and H.S. Reall, General supersymmetric AdS(5) black holes, J. High Energy Phys. 04 (2004) 048.

[24] H. K. Kunduri, J. Lucietti, and H. S. Reall, Supersymmetric multi-charge $\operatorname{AdS}(5)$ black holes, J. High Energy Phys. 04 (2006) 036.

[25] Z. Chong, M. Cvetic, H. Lu, and C. Pope, Five-dimensional gauged supergravity black holes with independent rotation parameters, Phys. Rev. D 72, 041901 (2005).

[26] A. Hanany, N. Mekareeya, and G. Torri, The Hilbert series of adjoint SQCD, Nucl. Phys. B825, 52 (2010).

[27] S. M. Hosseini, K. Hristov, and A. Zaffaroni, An extremization principle for the entropy of rotating BPS black holes in $\mathrm{AdS}_{5}$, J. High Energy Phys. 07 (2017) 106.

[28] S. Murthy, The growth of the $\frac{1}{16}$-BPS index in $4 \mathrm{~d} \mathcal{N}=4$ SYM, arXiv:2005.10843. 